\title{
Using the "flipped classroom" model in the teaching of the theoretical disciplines (French language) at the university
}

\section{[Technologie de la classe inversee dans l'enseignement des disciplines theoriques (FLE) aux etudiants des universites]}

\author{
Liliya R. Abdullina - Anastasia V. Ageeva - Natalia V. Gabdreeva
}

DOI: 10.18355/XL.2019.12.01XL.12

\begin{abstract}
This article aims to evaluate the introduction of a new technology of the "flipped classroom" in the process of education. A brief historical overview of the application of the flipped learning in the education system is provided. Approaches of leading supporters of this technology are considered. To prove its efficiency, we describe the results of our pedagogical experiment implemented on the course of the theoretical grammar of the French language with the students of Kazan Federal University. The work in both groups was carried out in three stages: knowledge assimilation, verification of the assimilation of resources and putting into practice. At each stage, similarities and differences in the compared groups are described. The experiment concludes with the final testing. The results of the experiment demonstrate the predominance of the experimental group over the control one. The factors explaining the expediency and efficiency of the application of the given technology in the course of theoretical disciplines in a foreign language at the University are formulated. Perspectives of future research of the technology of "flipped learning" are presented.
\end{abstract}

Key words: flipped classroom, language, student, independent work, effectiveness, technology

\section{Résumé}

L'article présent traite le problème de l'application de la classe inversée à l'enseignement linguistique des étudiants des universités. Après avoir donné les preuves à l'appui de son actualité nous présentons une brève historique de sa mise en pratique au système d'enseignement tout en examinant certaines approches des scientifiques adeptes de ladite technologie. Comme preuve de son éfficacité, nous décrivons les résultats de notre expérience pédagogique, réalisée dans le cadre du cours de la grammaire théorique de la langue française auprès des étudiants de l'Université Fédérale de Kazan. Le travail dans deux groupes d'étudiants est organisé en trois étapes: assimilation de connaissances, vérification de l'assimilation des ressources, mise en pratique. Nous décrivons les convergences et les divergences qui apparaissent entre ces deux groupes à chaque étape. L'expérience s'achève par un test final, il en découle que le groupe expérimental est plus performant que le groupe de contrôle. Nous dégageons les facteurs qui expliquent la pertinence et l'efficacité de l'application de la classe inversée à l'enseignement des disciplines théoriques en langue étrangère à l'Université. Pour conclure nous présentons certaines perspectives des recherches ultérieures consacrées à la technologie de la classe inversée.

Mots-clés: classe inversée, langue, étudiant, le travail autonome, efficacité, technologie

\section{Introduction}

Le milieu éducatif contemporain connaît des changements considérables qui se produisent sous l'influence de l'application des technologies d'information et de communication (TIC) au processus d'enseignement. En plus le 25 octobre 2016 le

XLinguae, Volume 12 Issue 1XL, January 2019, ISSN 1337-8384, eISSN 2453-711X 
Conseil du Développement Stratégique et des Projets Prioritaires auprès du Président de la Fédération de Russie a approuvé le programme "Le milieu contemporain de l'éducation numérique de la Fédération de Russie" visant à créer vers 2018 "les conditions pour améliorer la qualité et pour élargir les moyens de l'enseignement continu pour toutes les catégories de la population par la voie du développement de l'espace éducatif numérique en Russie et de l'augmentation du nombre d'étudiants qui ont maîtrisé le cursus en ligne jusqu'à 11 millions vers la fin de 2025" (2016). Par conséquent, les programmes en ligne / à distance jouissent d'une grande popularité en Russie ce qui favorise l'apparition de nouveaux termes, de nouvelles méthodes et technologies éducatives s'implantant d'une façon active dans les modes traditionnels de l'enseignement.

\section{La classe inversée comme approche pédagogique en enseignement}

\subsection{Concept de la classe inversée : aspect diachronique}

Parmi les approches innovatrices de la didactique des langues nous pouvons dégager la stratégie de la classe inversée (de l'anglais flipped classroom) qui prend de l'ampleur surtout à l'étranger (Le Jeune, 2016, Hung, 2017, Ayçiçek, Yelken, 2018, Lee, Wallace, 2018, Limniou, Schermbrucker, Lyons, 2018, Lopes, Soares, 2018, Mohamed, Lamia, 2018, Pellas, 2018). La conception de la classe inversée a été inventée il y a une dizaine d'années par Aaron Sams et Jonathan Bergmann, deux professeurs de chimie à Woodland Park High School (Etats-Unis, Colorado). En 2008 ils ont mis en ligne les premières vidéos de leurs cours destinées aux élèves de grandes classes. En même temps Salman Khan (2011), fondateur et directeur général de la Khan Academy, une des plus grandes écoles virtuelles, a proposé à son audience des tutoriels vidéo et, ensuite, une publication de son intervention à la TEDconférence où il avait appelé à réinventer l'enseignement. En 2012 paraît le livre de Sams et Bergmann intitulé Flip Your Classroom: Reaching Every Student in Every Class Every Day (2012) qui contribue à l'application du modèle de la classe inversée au système éducatif européen. Parmi les partisans dudit modèle on peut nommer surtout Marcel Lebrun, technopédagogue belge, professeur de l'Université catholique de Louvain, qui a exposé sa vision de la classe inversée en 2015 lors de la conférence vidéo Les classes inversées : enseigner à l'envers, apprendre à l'endroit ou l'inverse (2015). En France cette conception est soutenue par Héloïse Dufour, Présidente de l'association Inversons la Classe (ILC), ayant pour objet d'impulser et d'accompagner le développement et la mise en oeuvre des pratiques pédagogiques de classe inversée, et / ou centrées sur l'apprenant et/ou favorisant la motivation et la réussite de tous les apprenants, en particulier pour lutter contre les inégalités et le décrochage scolaire en France et dans le monde francophone, dans toutes les disciplines et à tous les niveaux d'apprentissage; d'impulser et d'accompagner, de réaliser des recherches universitaires et scientifiques sur les pratiques pédagogiques de la classe inversée.

\subsection{Différentes approches de la classe inversée}

Avant de passer à la descritpion des resultats de notre recherche il nous semble nécessaire de préciser la définition du terme "classe inversée". Marcel Lebrun partage la définition de l'Université Vanderbilt et qualifie ce terme comme suit:

"Le concept de classe inversée décrit un renversement de l'enseignement traditionnel. Les étudiants prennent connaissance de la matière en dehors de la classe, principalement au travers de lectures ou de vidéos. Le temps de la classe est alors consacré à un travail plus profond d'assimilation des connaissances au travers de méthodes pédagogiques comme la résolution de problèmes, les discussions ou les débats » (Brame, 2013). 
On peut voir une autre définition dans l'ouvrage de Héloïse Dufour :

"Il s'agit d'une inversion spatiale et temporelle par rapport à la classe traditionnelle, où l'enseignant transmet son savoir sous la forme d'un cours magistral ou dialogué en classe, avant de faire réaliser aux élèves des exercices d'application et d'approfondissement dont l'exécution est souvent reléguée hors la classe par manque de temps.» (Dufour, $2014: 44$ ).

Le chercheur russe E.Voulfovitch définit le modèle du flipped classroom de la manière suivante :

«La maîtrise autonome de la matière à enseigner que les apprenants réalisent hors classe, généralement sous la forme de cours video, suivie d'une discussion détaillée et $d u$ transfert des connaissances dans une nouvelle situation: résolution d'un problème pratique, souvent de caractère créateur, qui entend une discussion, un jeu de rôle, un projet ou un cas, une quête web, etc. Ce modèle inverse le travail en classe et le devoir à domicile. » (Vulfovich, 2017: 89).

En plus le modèle du flipped classroom vise à l'intégration des TIC dans le processus didactique pour l'améliorer et pour motiver les apprenants. Il est évident que la classe inversée peut utiliser toute sorte de matériel vidéo: des cours, des webinaires, des vidéographies et des présentations. L'utilisation des TIC permet d'intensifier le travail autonome des étudiants, car elle correspond à des besoins, des connaissances et des acquis des enfants appartenant à la génération $Z$.

Il est évident que toute approche de la classe invérsée est basée sur trois principes déterminés par Héloise Dufour comme suit : l'assimilation de connaissances (hors la classe), la vérification de l'assimilation des ressources, la mise en pratique (l'utilisation des ressources) dans la classe (Dufour, 2014 : 45). Provoquant beaucoup de discussions, ils sont experimentés dans les cours variés à des différents niveaux d'enseignement. Pourtant à l'heure actuelle l'application de la classe inversée dans le cours de langue étrangère n'est pas assez étudiée, ce qui nous a poussé de lancer une expérience pédagogique afin de démontrer sa pertinence dans les cours théoriques du français.

\section{Méthodologie de recherche}

\subsection{Méthodes de recherche}

L'étude est basée sur la variété des méthodes, parmi lesquelles se distinguent :

- l'analyse didactique des pratiques d'enseignement;

- l'analyse systémique des usages des technologies de l'information et de la communication pour l'enseignement-apprentissage du FLE dans l'université;

- le recours à des questionnaires et des tests;

- l'expérience pédagogique menée avec des étudiants de deuxième année en "Traduction/interprétation et Traductologie/Interpretariat" à l'Institut des relations internationales de l'Université Fédérale de Kazan.

- la comparaison des résultats de l'expérience.

\subsection{Questions de recherche}

I. Le modèle de la clase inversée a été appliqué dans le cours de la grammaire théorique de la langue française destiné aux étudiants de la $2^{\text {ème }}$ année spécialisés en linguistique ("Traduction/interprétation et Traductologie/Interpretariat") à l'Ecole Supérieure des Langues Etrangères et de la Traduction/Interpretation de l'Université Fédérale de Kazan (Russie). Le programme d'étude prévoit 24 heures de cours XLinguae, Volume 12 Issue 1XL, January 2019, ISSN 1337-8384, eISSN 2453-711X 
théoriques, 12 heures de travaux pratiques et 54 heures de travail autonome. Les cours sont donnés en français car les étudiants l'apprennent comme première langue étrangère. La tâche de comprendre les détails de la grammaire théorique en classe étant assez difficile en $2^{\text {ème }}$ année, nous avons décidé d'avoir recours à la technologie de la classe inversée. L'objectif de cette expérience était de justifier l'éfficacité du modèle éducatif de la classe inversée dans l'enseignement des disciplines théoriques en langue étrangère. Parmi les tâches primordiales de la recherche nous avons défini l'augmentation de l'intérêt des étudiants au matériel didactique et le développement de leur compétence communicative.

Nous avons proposé aux étudiants de deux groupes le matériel didactique identique (Unité 2. Parties du discours). L'enseignement dans le groupe de contrôle a été organisé d'une façon traditionnelle, tandis que le groupe expérimental a suivi le modèle de la classe inversée. Les divergences ont été enregistrées à chaque étape:

I. Assimilation de connaissances - travail des étudiants avant le cours (hors classe)

Dans le groupe de contrôle cette étape est absente parce que les étudiants ne font aucun travail avant le cours. Les étudiants du groupe expérimental, au contraire, ont à faire une série de devoirs suivant les instructions du professeur:

1. Voir le cours vidéo (10 minutes de durée) relevant de la ressource d'apprentissage électronique Grammaire théorique (https://edu.kpfu.ru/course/view.php?id=2248) mis en ligne dans le cadre du système e-learning de l'Université Fédérale de Kazan.

2. Faire des exercices visant à comprendre les savoirs théoriques et présentés dans l'unité 2 "Parties du discours" de la même ressource. Les exemples des exercices sont cités ci-dessous.

\begin{tabular}{|c|c|}
\hline \multicolumn{2}{|l|}{ 1. Choisissez la bonne réponse : } \\
\hline Les parties du discours subsidiaires sont & $\begin{array}{l}\text { a) le substantif; } \\
\text { b) le verbe; } \\
\text { c) l'adjectif; } \\
\text { d) l'adverbe; } \\
\text { e) la conjonction; } \\
\text { f) la préposition; } \\
\text { g) l'interjection. }\end{array}$ \\
\hline \multicolumn{2}{|l|}{ 2. Associez: } \\
\hline $\begin{array}{l}\text { 1 La préposition } \\
2 \text { L'interjection } \\
3 \text { La particule } \\
4 \text { La conjonction }\end{array}$ & $\begin{array}{l}\text { a) Le mot autonome à valeur } \\
\text { expressive; } \\
\text { b) Le mot-outil ; } \\
\text { c) Le morphème grammatial ; } \\
\text { d) Le mot autonome à valeur } \\
\text { nominative. }\end{array}$ \\
\hline \multicolumn{2}{|c|}{ 3. Déterminez si les affirmations suivantes sont vraies ou fausses. } \\
\hline 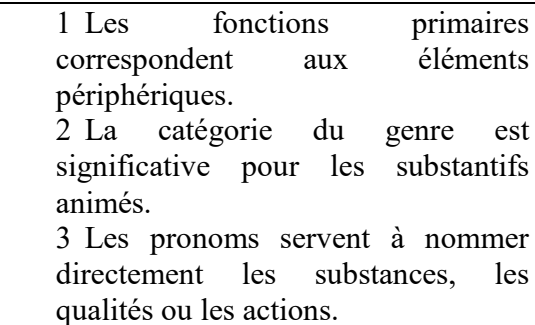 & $\begin{array}{l}\text { a) Vrai } \\
\text { b) Faux }\end{array}$ \\
\hline
\end{tabular}

\section{Tableau 1}

Exercices visant à comprendre le matériel théorique 
3. Répondre aux questions. La discussion avec le professeur prévoit une liste de questions auxquelles les étudiants doivent répondre au préalable en effectuant la recherche et l'analyse autonomes.

La première série de questions porte sur la maitrise des savoirs théoriques. Par ex.:

a Trouvez la signification de terme «Partie du discours». Comparez différentes approches linguistiques.

b Dégagez les parties du discours essentielles et subsidiaires. Évaluez leurs traits pertinents.

c Expliquez la divergence d'opinions des linguistes concernant le nombre des parties du discours. Déterminez les critères mis en base d'analyse.

La deuxième série de questions permet d'évaluer les savoir-faire et les compétences pratiques grâce à l'analyse des exemples concrets en français. Citons quelques exemples.

1. Quel substantif assume la fonction secondaire dans le groupe de mot suivant ?

a) boîte en papier;

b) boîte en papier;

c) boîte en papier.

2. Déterminez le type de la transposition grand (adj.) $\rightarrow$ un grand $(n)$ :

a) morphologique (affixation);

b) morphologique (conversion);

c) syntaxique.

3. Quels sont les traits communs et distinctifs des unités lexicales suivantes :

marin, $m$ - homme de mer; soigner - prendre soin, donner soin; militaire, $m$ - homme de guerre; ecclésiastique, $m$ - homme d'église; visiter - faire visite; hâte, à la hâte; doucement - en douce, à la douce; aveuglement - à l'aveuglette; ménagère, $f$-femme de ménage; soudain - tout à coup; mouvementer - mettre en mouvement.

II. Vérification de l'assimilation des ressources - travail des étudiants en classe.

Le groupe de contrôle suit le cours donné par le professeur et accompagné d'une présentation numérique.

Le groupe expérimental participe à la discussion animée par le professeur: les étudiants discutent le cours vidéo vu hors classe, le professeur révèle les moments difficiles à comprendre à la base des questions qu'ils posent et de l'analyse des résultats de leur travail virtuel. Il faut noter que la ressource d'apprentissage électronique prévoit l'examen pas à pas des travaux des étudiants et l'évaluation de leurs succès et de leurs échecs par l'auteur / professeur ce qui permet de choisir les exercices supplémentaires pour la meilleure assimilation des connaissances.

III. Mise en pratique - travail des étudiants après le cours (en et hors classe).

La classe traditionnelle suppose la distribution des variantes imprimées des exercices servant à assimiler les savoirs théoriques et leur révision pendant les travaux pratiques. Les travaux pratiques s'achèvent par un test fait en classe. Les résultats sont mis en lumière après leur examen par le professeur, habituellement pendant la leçon suivante. En outre, les apprenants écrivent des essais où ils analysent de différentes approches des scientifiques qui ont consacré leurs ouvrages à des bases théoriques de la grammaire française en fonction du sujet étudié.

Les apprenants du groupe expérimental préparent un essai numérique qu'ils envoient au professeur sous forme de fichiers joints en utilisant le système universitaire elearning. Le professeur y évalue les travaux et donne des commentaires. Finalement les étudiants font un test qui a ses délais et sa durée fixes, la limite du temps servant à exclure la recherche possible des réponses à d'autres ressourses informatiques. Les résultats sont évalués immédiatement par le système. Les réponses incorrectes sont accompagnées de commentaires.

Ainsi, le contenu de l'expérience prévoit les devoirs identiques dans le groupe de 
contrôle et celui expérimental, imprimés ou numériques respectivement.

\section{Résultats et discussion}

A l'étape initiale pour assurer l'objectivité de l'expérience nous avons testé le niveau de français des apprenants dans les deux groupes, conformément aux exigences du cursus : 1-10 réponses correctes - niveau bas, 11-15 réponses correctes - niveau moyen, 16-20 reponses correctes - niveau élevé.

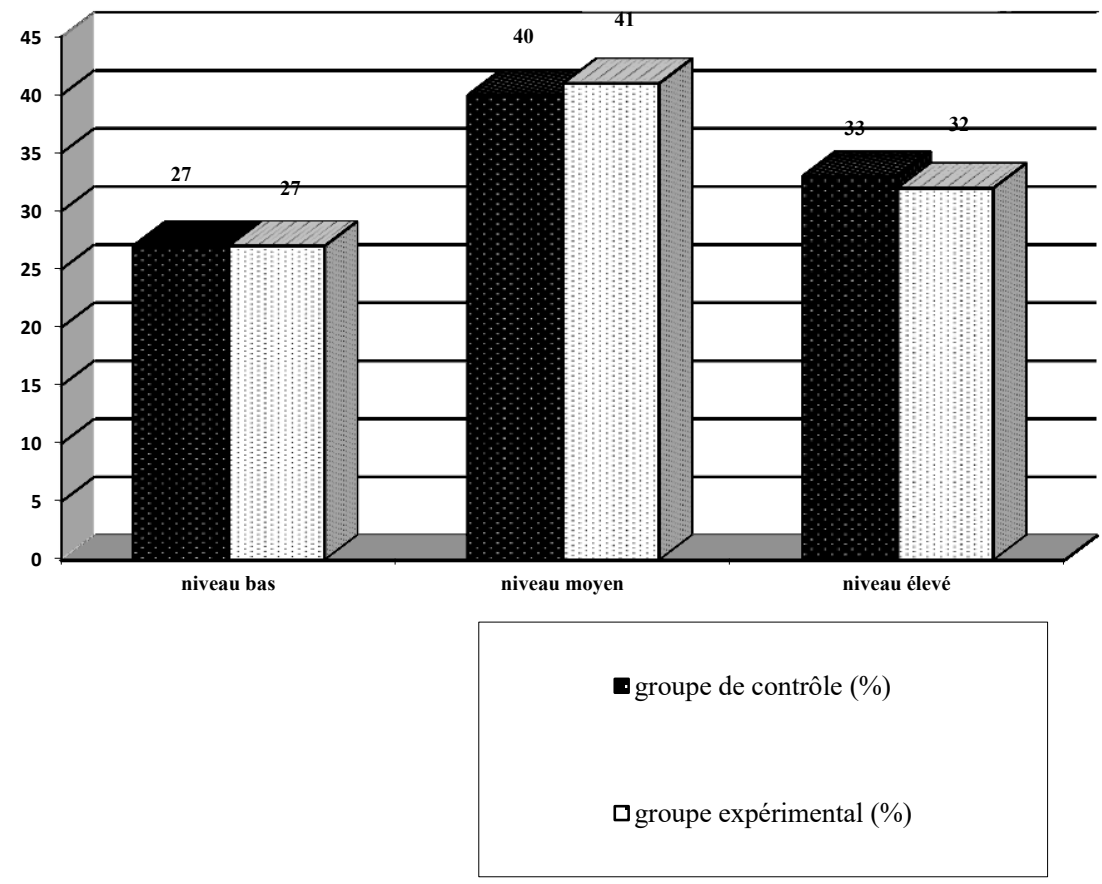

\section{Graphique 1}

Niveau de la maîtrise de la langue française

Le graphique 1 montre que le niveau de français des étudiants était presque identique ce qui nous a permis de passer à l'expérience.

Cependant les résultats du test final ont montré à leur tour les divergences en matière de l'assimilation des connaissances. Nous avons dégagé 4 niveaux de la maîtrise des savoirs (une réponse correcte est evaluée à 1 point, le maximum atteint 20 points):

insatisfaisant - moins de 5 points;

satisfaisant - de 5 à 10 points;

bon - de 11 à 15 points;

excellent - de 16 à 20 points. 


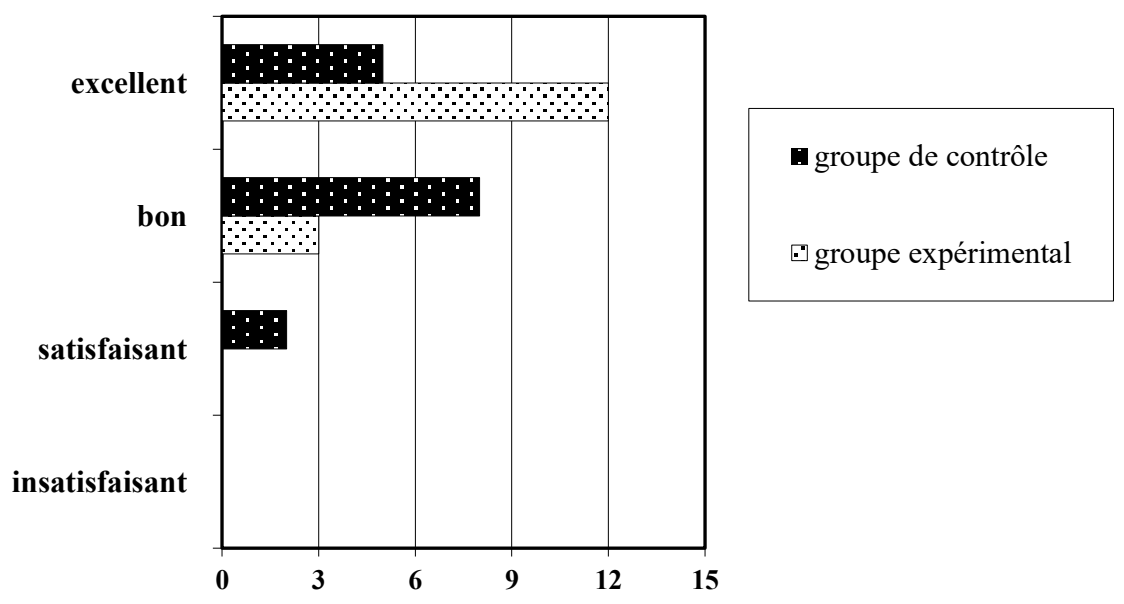

\section{Graphique 2}

Résultats du test final

Le graphique 2 met en évidence que dans le groupe expérimental 12 étudiants sur 15 ont montré un niveau excellent de la maîtrise des savoirs tandis que dans le groupe de contrôle ce niveau n'a été atteint que par 5 apprenants. Plusieurs étudiants du groupe de contrôle ont obtenu de 10 à 15 points, dans le groupe expérimental 3 apprenants ont montré les résultats pareils. Le niveau satisfaisant n'a été enregistré que dans le groupe de contrôle -2 étudiants.

Ces données nous permettent de constater l'efficacité de la technologie de la classe inversée dans le cadre de l'apprentissage de la grammaire théorique de la langue française. Ce fait s'explique évidemment par plusieurs facteurs :

1. A l'étape de l'assimilation les apprenants du groupe expérimental ont l'occasion d'étudier le matériel en détail, de revoir la vidéo, de se servir de dictionnaires et de faire des exercices d'entraînement. Les étudiants du groupe de contrôle sont privés de la possibilité de le faire.

2. A l'étape de la vérification l'activité cognitive des étudiants du groupe expérimental s'intensifie, ils sont plongés dans la discussion, ils sont capables de résoudre les cas et d'analyser les problèmes pratiques complexes. Dans le groupe de contrôle les étudiants reçoivent l'information passivement, ils ont souvent du mal à comprendre le cours à cause des connaissances linguistiques insuffisantes. En plus, un étudiant ne peut pas interrompre le professeur pour lui demander de répéter et d'expliquer chaque fois où il n'a pas entendu ou n'a pas compris le contenu de l'énoncé. Il n'aura cette occasion que pendant la préparation à l'étape suivante.

3. L'étape de la mise en pratique dans le groupe expérimental prévoit le travail à distance visant plutôt à contrôler les connaissances acquises. Le groupe de contrôle travaille en classe ce qui suppose l'exercice pratique et le test immédiat organisé à son issue. Malheureusement les heures de classe sont limitées et il est impossible de consacrer trois leçons à un sujet. Alors, comme l'acquisition des connaissances dans le groupe de contrôle est plus lente, les résultats du test final sont pires.

\section{Conclusion}

Ainsi, dans la situation où les heures du travail autonome des étudiants des universités XLinguae, Volume 12 Issue 1XL, January 2019, ISSN 1337-8384, eISSN 2453-711X 
augmentent tandis que celles en classe sont diminuées, la technologie de la classe inversée peut être une des méthodes efficaces de l'enseignement des disciplines théoriques en langue étrangère : elle permet une économie considérable du temps, ainsi que l'intensification et le contrôle permanent des activités des apprenants hors classe, le développement de la parole et de la pensée en langue étrangère tout en créant les conditions de la formation et de l'évolution de leurs compétences informationnelles et communicatives.

Sans aucun doute, les résultats de notre recherche ne déprécient pas les formes traditionnelles de l'enseignement et demandent des approbations plus longues et plus détaillées, à la base des autres disciplines linguistiques. Il s'en suit que les perspectives des recherches pareilles sont multiples : il serait intéressant de définir les possibilités et les conditions de l'application de la classe inversée dans la pratique de l'enseignement des langues et des cultures linguistiques ou d'effectuer une analyse complète de ladite technologie compte rendu de ses avantages et de ses inconvénients du point de vue des apprenants et des enseignants.

\section{Acknowledgments}

The work is performed according to the Russian Government Program of Competitive Growth of Kazan Federal University.

\section{Bibliographic references}

AYÇIÇEK, B. - YELKEN, T.Y. 2018. The effect of flipped classroom model on students' classroom engagement in teaching English. In: International Journal of Instruction, vol. 11, n. 2, pp. 385-398. DOI: 10.12973/iji.2018.11226a

BERGMANN, J. - SAMS, A. 2012. Flip Your Classroom: Reach Every Student in Every Class Every Day. ISTE. ISBN 978-1-56484-315-9

BRAME, C. 2013. Flipping the classroom. Vanderbilt University Center for Teaching. Available online: http://cft.vanderbilt.edu/guides-sub-pages/flipping-the-classroom/ DUFOUR, H. 2014. La classe inversee. In : Technologie, n. 193, pp. 44-47. Available online:

http://eduscol.education.fr/sti/sites/eduscol.education.fr.sti/files/ressources/techniques/ 6508/6508-193-p44.pdf

HUNG, H.-T. 2017. Language teaching and technology forum: The integration of a student response system in flipped classrooms. In: Language Learning and Technology, vol. 21, n. 1, pp. 16-27. ISSN: 10943501

KHAN, S. 2011. Let's use video to reinvent education. Available online: https://www.youtube.com/watch?v=nTFEUsudhfs

LEBRUN, M. 2015. Les classes inversees: enseigner a l'envers, apprendre a l'endroit ou l'inverse? Available online: https://videos.univlorraine.fr/index.php?act=view\&id=2176

LEE, G. - WALLACE, A. 2018. Flipped Learning in the English as a Foreign Language Classroom: Outcomes and Perceptions. In: Tesol quarterly, vol. 52, n. 1, pp. 62-84. DOI: $10.1002 /$ tesq.372

LE JEUNE, J.-M. 2016. Flipped classroom: Didactic triangle meaning above below [La classe inversée: Le triangle pedagogique sens dessus dessous]. In : Synergies Turquie, vol. 9, pp. 161-172. ISSN: 19619472

LIMNIOU, M. - SCHERMBRUCKER, I. - LYONS, M. 2018. Traditional and flipped classroom approaches delivered by two different teachers: the student perspective. In: Education and information technologies, vol. 23, n. 2, pp. 797-817. DOI: $10.1007 / \mathrm{s} 10639-017-9636-8$

LOPES, A.P. - SOARES, F. 2018. Perception and performance in a flipped Financial Mathematics classroom. In: International Journal of Management Education, vol. 16, n. 1, pp. 105-113. DOI: 10.1016/j.ijme.2018.01.001

MOHAMED, H. - LAMIA, M. 2018. Implementing flipped classroom that used an 
intelligent tutoring system into learning process. In: Computers and Education, vol. 124, pp. 62-76. DOI: 10.1016/j.compedu.2018.05.011

PELLAS, N. 2018. Is the flipped classroom model for all? Correspondence analysis from trainee instructional media designers. In: Education and information technologies, vol. 23, n. 2, pp. 757-775. DOI: 10.1007/s10639-017-9634-x

PRIORITETNYJ PROEKT. 2016. Sovremennaya tsifrovaya obrazovatel'naya sreda v Rossijskoj Federatsii. Available online: http://static.government.ru/media/files/8SiLmMBgjAN89vZbUUtmuF51ZYfTvOAG. pdf

VULFOVICH, E.V. 2017. Flipped classroom for organization of EFL studentsindependent work. In: Education in Russia, n. 4. pp. 88-95. ISSN 0869-3617

Words: 3606

Characters: 24481 (13,6 standard pages)

Assoc. prof. Liliya R. Abdullina, PhD

Institute of International Relations

Kazan (Volga region) Federal University

18 Kremlyovskaya Str.

420008 Kazan

Russia

lilioven@mail.ru

Assoc. prof. Anastasia V. Ageeva, PhD

Institute of International Relations

Kazan (Volga region) Federal University

18 Kremlyovskaya Str.

420008 Kazan

Russia

anastasia_ageeva@mail.ru

Academician Natalia V. Gabdreeva, Dr. Habil.

Kazan National Research Technical University named after A. N. Tupolev - KAI

10 K. Marx Str.

420111 Kazan

Russia

n.gabdreeva@mail.ru

XLinguae, Volume 12 Issue 1XL, January 2019, ISSN 1337-8384, eISSN 2453-711X 